\title{
Turbulencia sintética tridimensional: escalamiento anómalo en el rango inercial y propiedades multifractales de la disipación
}

\author{
Three-dimensional synthetic turbulence: anomalous scaling in the inertial \\ range and multifractal properties of the dissipation
}

Carlos Rosales H. ${ }^{1}$

Recibido 11 de junio de 2009, aceptado 13 de abril de 2011

Received: June 11, 2009 Accepted: April 13, 2011

\begin{abstract}
RESUMEN
Este trabajo presenta un análisis de las propiedades de escalamiento del método MMLM, utilizado para la construcción numérica de campos vectoriales turbulentos sintéticos tridimensionales. En particular, se estudian las propiedades de escala para las funciones de estructura del campo de velocidades, encontrándose que el MMLM conduce a un escalamiento del tipo Kolmogorov. Si el parámetro de mapeo, consistente en una escala de tiempo basada en la separación de nodos sobre la malla computacional, es modificado y asimilado al tiempo característico de evolución de los vórtices para cada escala espacial, se observa que el campo de turbulencia sintética presenta el escalamiento anómalo propio de los campos turbulentos reales, con muy buena concordancia respecto a los valores conocidos. Adicionalmente se estudian la intermitencia y naturaleza multifractal de la distribución de disipación de energía. Los resultados son también consecuentes con las observaciones en turbulencia real. El estudio arroja nueva luz sobre cuáles son los mínimos requerimientos dinámicos para obtener escalamiento anómalo en el rango inercial.
\end{abstract}

Palabras clave: Turbulencia, intermitencia, simulación numérica, multifractales, modelos estocásticos.

\section{ABSTRACT}

This work presents an analysis of the scaling properties of the MMLM method, used for the numerical construction of three-dimensional turbulent vector fields. Specifically, the scaling properties for the velocity field structure functions are studied. It is found that MMLM gives scaling of Kolmorogov type. If the mapping parameter, which is given by a time scale based on node separation over the computational mesh, is modified and equated to the characteristic time for eddy evolution at each spatial scale, the synthetic turbulent field presents the characteristic anomalous scaling of real turbulent fields, with very good agreement with respect to the known values. In addition, we study the intermittency and multifractal nature of the energy dissipation distribution. Results are also consistent with observations in real turbulence. The study sheds new light over the minimal dynamical requirements to obtain anomalous scaling in the inertial range.

Keywords: Turbulence, intermittency, numerical simulation, multifractals, stochastic models.

\section{INTRODUCCIÓN}

Se entiende por turbulencia sintética la construcción de campos de variables que presentan características propias de fluctuaciones turbulentas, pero que puedan obtenerse con un reducido costo computacional en comparación con una resolución numérica de las ecuaciones de Navier-Stokes. En este sentido, la turbulencia sintética substituye, en mayor o menor medida, la dinámica real del fenómeno por procesos más simples, orientados a reproducir las características observables. Un nuevo procedimiento

1 Departamento de Ingeniería Mecánica, Universidad Técnica Federico Santa María, Casilla 110-V, Valparaíso, Chile. E-mail: carlos.rosales@usm.cl 
para generar campos vectoriales turbulentos sintéticos, llamado MMLM (Minimal Multiscale Lagrangian Map), fue introducido en [1], observándose que este método es capaz de reproducir muchas de las propiedades fundamentales de la turbulencia, tales como el sesgo y curtosis de los gradientes de velocidad, la forma general de las funciones de densidad de probabilidad (PDF) para derivadas e incrementos de velocidad, la PDF conjunta del segundo y tercer invariantes del tensor gradiente de velocidad, el alineamiento de la vorticidad con el eigenvector intermedio del tensor de deformación, y otras características concernientes a la producción de enstrofía y tasa de deformación.

Se definen los incrementos longitudinales de velocidad sobre un vector de separación $\mathbf{r}$ como

$$
\delta u_{\|}(r)=[\mathbf{u}(\mathbf{x}+\mathbf{r})-\mathbf{u}(\mathbf{x})] \cdot \mathbf{r} / r
$$

Se observó en [1] que la estadística de las funciones $\delta u_{\|}(r)$ resultantes dependía de la escala espacial $r$, con curtosis que se incrementa progresivamente al reducir la escala, lo cual indica la presencia de intermitencia en el campo de velocidad, en el rango inercial de escalas. Esta intermitencia en el rango inercial es un tópico de primera importancia en la teoría moderna de turbulencia, puesto que representa una desviación de la invariancia estadística de escalas que subyace en la teoría clásica de Kolmogorov (K41). Esta invariancia se traduce esencialmente en que a escalas $r$ suficientemente pequeñas (con respecto a la escala integral de la turbulencia, $L$ ), el flujo es autosimilar en el sentido de que existe un único exponente $\beta$ de escalamiento tal que

$$
\delta u_{\|}(\varphi r) \stackrel{\text { ley }}{\longleftrightarrow} \varphi^{\beta} \delta u_{\|}(r)
$$

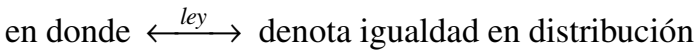
estadística, y $\varphi$ es alguna constante (tal que $\varphi r \ll<$ ). Un resultado fundamental en turbulencia homogénea isotrópica es la "ley 4/5" de Kolmogorov, para la función longitudinal de estructura de tercer orden [2],

$$
\left\langle\left[\delta u_{\|}(r)\right]^{3}\right\rangle=-\frac{4}{5} \varepsilon r, \text { cuando } \eta \ll r<<L
$$

donde $\varepsilon$ es la razón media de disipación de energía cinética, $\eta$ es la microescala de Kolmogorov y el operador $\langle\cdot\rangle$ indica media estadística. Esta expresión puede derivarse rigurosamente de la ecuación de Navier-Stokes y es asintóticamente exacta para elevados números de Reynolds. La ecuación (3) implica obviamente que

$$
\left\langle\left[\delta u_{\|}(\varphi r)\right]^{3}\right\rangle=\varphi\left\langle\left[\delta u_{\|}(r)\right]^{3}\right\rangle
$$

mientras que la ecuación (2) implica un valor $\varphi^{3} \beta$ para el prefactor, de modo que el exponente de escalamiento debe $\operatorname{ser} \beta=1 / 3$. Una generalización de este resultado, basada en argumentos dimensionales, conduce entonces al escalamiento de la función de estructura de orden $p$ como una ley de potencia:

$$
\left\langle\left[\delta u_{\|}(r)\right]^{p}\right\rangle \propto r^{\zeta_{p}}
$$

con $\zeta_{p}=p / 3$ cuando el campo de velocidad posee estadísticamente la invariancia de escalas implícita en la teoría K41. No obstante, se ha acumulado mucha evidencia experimental y numérica $[3,4]$ de que los flujos turbulentos presentan intermitencia en el rango inercial, y el escalamiento es anómalo con respecto a lo predicho en el párrafo que antecede. Esta anomalía se manifiesta por una desviación de los exponentes $\zeta_{p}$ del valor $p / 3$ derivado para el caso no intermitente, tornándose una función no-lineal (cóncava no-decreciente) del orden $p$. El volumen de investigación sobre este tópico es sumamente extenso para una revisión aquí. Se recomienda a lector consultar [4] y las referencias que allí aparecen.

En el método MMLM no se utiliza ningún modelo estadístico a priori para introducir intermitencia, de modo que es de interés determinar si el escalamiento anómalo puede surgir incluso bajo la dinámica sumamente simplificada en la que este método se basa. Por lo tanto, el objetivo principal de este trabajo es determinar si el MMLM es capaz de reproducir también la intermitencia en el rango inercial. Adicionalmente se busca estudiar y caracterizar el campo de disipación de energía resultante del proceso de síntesis numérica de la velocidad. 


\section{MÉTODO MMLM}

El MMLM se describe en detalle en [1]; aquí sólo se dará una descripción muy resumida. La idea básica es deformar un campo de velocidades aleatorio inicialmente gaussiano, mediante una función de mapeo de las velocidades hacia nuevas posiciones, como si fuesen transportadas por partículas fluidas no interactuantes. Cuando toda interacción entre partículas es suprimida, la ecuación de movimiento se reduce a la ecuación de Riemann

$$
\partial_{t} \mathbf{u}+(\mathbf{u} \cdot \nabla) \mathbf{u}=0
$$

cuya solución es $\mathbf{u}(\mathbf{X}(t), t)=\mathbf{u}(\mathbf{x}, 0)$, donde $\mathbf{X}(t)$ es la posición lagrangiana de la partícula adscrita en $t=0$ a la posición euleriana $\mathbf{x}$ :

$$
\mathbf{X}(t)=\mathbf{x}+t \mathbf{u}(\mathbf{x}, 0) .
$$

De este modo, la deformación consiste esencialmente en la autoadvección del campo vectorial de velocidades. Para esto, el método procede mediante una descomposición multiescala para diferentes niveles $n$ o escalas espaciales $\ell_{n}=2^{-n} \ell_{0}$, con $\{n=1, \ldots, M\}$, siendo la escala inicial $\ell_{0}$ del orden de la escala integral $L$. La descomposición de la velocidad se puede escribir como

$$
\mathbf{u}=\mathbf{u}_{n}^{<}+\mathbf{u}_{n}^{>}
$$

la cual emplea un filtro cut-off en el espacio de Fourier, a un número de onda $k_{c, n}=\pi / \ell_{n}$. El campo $\mathbf{u}_{n}^{<}$se obtiene mediante un filtrado pasa-bajos de las velocidades generadas en el nivel previo $(n-1)$. Para cada escala, este campo es representado en el espacio físico sobre una malla que utiliza la escala $\ell_{n}$ como espaciamiento entre nodos. Empezando a la mayor escala disponible $(n=1)$, el MMLM distorsiona la porción de gran escala de dicho campo mediante el mapeo definido por la ecuación (7):

$$
\mathbf{X}=\mathbf{x}+t_{n} \mathbf{u}_{n}^{<}(\mathbf{x}), \quad \mathrm{y} \quad \mathbf{v}_{n}^{<}(\mathbf{X}) \equiv \mathbf{u}_{n}^{<}(\mathbf{x})
$$

El parámetro de mapeo, $t_{n}$, que cuantifica la extensión de la deformación, es determinado como

$$
t_{n}=\frac{\ell_{n}}{u_{\mathrm{rms}, n}^{<}}
$$

donde $u_{\text {rms, } n}^{<}$es la velocidad cuadrática media (r.m.s.) para el campo filtrado a escala $\ell_{n}$. Nuevas velocidades $\mathbf{v}_{n}^{<}(\mathbf{x})$ en las posiciones eulerianas $\mathbf{x}$ se obtienen a partir del campo de velocidades distorsionado (el cual reside ahora sobre una malla deformada no uniforme) mediante interpolación. Se interpola sobre las velocidades de las partículas que han arribado a la vecindad de $\mathbf{x}$ después de ser desplazadas de acuerdo a la función (9). La interpolación utiliza un promedio ponderado simple, empleando el inverso de la distancia $|\mathbf{x}-\mathbf{X}|$ como función de ponderación sobre una esfera de radio $\ell_{n}$ centrada en $\mathbf{x}$ :

$\mathbf{v}_{n}^{<}(\mathbf{x})=\sum_{|\mathbf{x}-\mathbf{X}|<\ell_{n}}|\mathbf{x}-\mathbf{X}|^{-1} \mathbf{v}_{n}^{<}(\mathbf{X}) / \sum_{|\mathbf{x}-\mathbf{X}|<\ell_{n}}|\mathbf{x}-\mathbf{X}|^{-1}$

Se consigue que el nuevo campo de velocidad $\mathbf{v}_{n}^{<}(\mathbf{x})$ sea solenoidal proyectándolo, en el espacio de Fourier, sobre el subespacio no divergente:

$$
\hat{\mathbf{w}}_{n}^{<}(\mathbf{k})=\mathbf{P}(\mathbf{k}) \cdot \hat{\mathbf{v}}_{n}^{<}(\mathbf{k})
$$

Aquí, el superíndice ${ }^{\wedge}$ denota cantidades Fouriertransformadas, y $\mathbf{P}(\mathbf{k})$ es el tensor de proyección en el espacio de Fourier sobre un plano ortogonal al vector de número de onda $\mathbf{k}$,

$$
P_{i j}=\delta_{i j}-k_{i} k_{j} / k^{2}
$$

siendo $\delta_{i j}$ el delta de Kronecker.

Las amplitudes de los modos de Fourier $\hat{\mathbf{w}}_{n}^{<}$son además renormalizadas de modo de satisfacer un espectro de energía $E(k)$ prescrito, según

$$
\hat{\mathbf{z}}_{n}^{<}(\mathbf{k})=\hat{\mathbf{w}}_{n}^{<}(k)\left\{\frac{E(k)}{\frac{1}{2} \sum_{|q|=k} \hat{\mathbf{w}}_{n}^{<}(\mathbf{q}) \cdot \hat{\mathbf{w}}_{n}^{<*}(\mathbf{q})}\right\}^{1 / 2}
$$

(* indica el complejo conjugado).

Este campo de gran escala es ahora combinado con la porción filtrada pasa-altos de la velocidad, $\mathbf{u}_{n}^{>}$, la cual ha permanecido inalterada a este nivel: $\mathbf{u}=\mathbf{z}_{n}^{<}+\mathbf{u}_{n}^{>}$. A continuación, el campo resultante $\mathbf{u}$ es tomado como el punto de partida para avanzar al siguiente nivel, y el filtrado (ahora a un cut-off 
más alto) y las demás operaciones se aplican una vez más con $n \leftarrow n+1$. De este modo la acción distorsionadora avanza hacia escalas progresivamente menores, hasta alcanzar una escala del orden de la microescala de Kolmogorov $\eta$. Al final de este proceso los efectos del mapeo lagrangiano se han superpuesto y acumulado sobre un rango de escalas espaciales, que abarca desde la escala integral hasta las escalas disipativas de energía.

\section{FUNCIONES DE ESTRUCTURA PARA EL CAMPO MMLM}

En primer lugar, el método MMLM se usó para generar campos de velocidad sintéticos sobre una malla de $N_{M}^{3}=512^{3}$ puntos en un dominio de tamaño $(2 \pi)^{3}$ con contornos periódicos. La escala más pequeña está dada por la separación $\ell_{M}=2 s N_{M}$ entre nodos, mientras que las escalas mayores están representadas por conjuntos reducidos de puntos, $N_{n}=N_{M} / 2^{M-n}$ para cada nivel $n=1, \ldots, M$. El espectro de energía prescrito es

$$
E(k)=C \varepsilon^{2 / 3} k^{-5 / 3} \exp \left(-\beta\left[(k \eta)^{4}+c_{\eta}^{4}\right]^{1 / 4}-c_{\eta}\right)
$$

con $C=1.5, \beta=5.2$ y $c_{\eta}=0.4$. Los parámetros $u_{\mathrm{rms}}$, $\varepsilon$ y la viscosidad $v$ son tales que el número de Reynolds (a escala de Taylor) es $\operatorname{Re}_{\lambda}=200$. El MMLM es aplicado empezando desde una malla gruesa con $N_{1}^{3}=16^{3}$ nodos, y la secuencia es $16^{3} \rightarrow 32^{3} \rightarrow 64^{3}$
$128^{3}-256^{3}-512^{3}$, es decir, $M=6$. El interés aquí se centra en las propiedades de escalamiento de las funciones de estructura. La Figura 1 muestra las funciones de estructura para diversos órdenes $p$. Las funciones tienden hacia un comportamiento de ley de potencia aproximado, pero solo sobre un rango limitado de longitudes $r 17 \ell_{M}<r<100 \ell_{M}$ ), el cual corresponde aproximadamente a $25 \eta<r<150 \eta$ (ya que $\ell_{M}=1,5 \eta$ ). Para la región donde las pendientes permanecen aproximadamente constantes, dichas pendientes se sitúan próximas al escalamiento de Kolmogorov (pendientes K41 se indican con líneas segmentadas).

Con el fin de elucidar si este comportamiento cambia en función de la razón de escalas disponibles, el procedimiento es repetido para un dominio con $1.024^{3}$ puntos $\left(N_{1}^{3}=16^{3} ; M=7\right)$. En este caso se tiene $\operatorname{Re}_{\lambda}=476$. Los resultados son presentados en la Figura 2 como líneas llenas (para mejor visualización los rangos con $\log _{10}\left(r / \ell_{M}\right)$ menor que 0,5 y mayor que 2,5 se han excluido, ya que se sitúan fuera del rango inercial). Se observa una mejor aproximación a una ley de potencia, pero con exponentes que no difieren significativamente de los predichos por la teoría K41. Téngase presente que estos campos sintéticos aún exhiben todas las características turbulentas mencionadas previamente (sesgo y PDF de los gradientes de velocidad, estadísticas para enstrofía y producción de deformación, etc.). Sin embargo, es claro que no hay escalamiento anómalo.
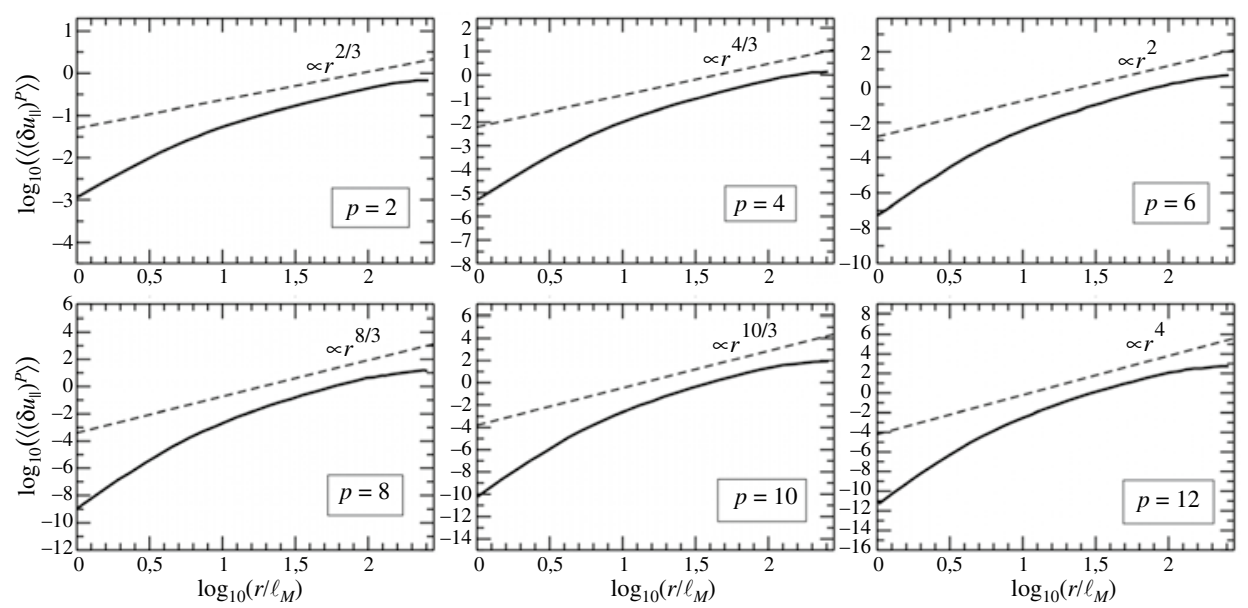

Figura 1. Funciones de estructura de velocidad para un campo MMLM de $512^{3}$ puntos. Las líneas segmentadas corresponden a la pendiente para escalamiento de Kolmogorov. 
La porción energética de gran escala del espectro no se incluye en la función (13), para extender el rango inercial tanto como sea posible. Para descartar la posibilidad de que el comportamiento observado esté asociado de algún modo con una falta de características realistas en la turbulencia de gran escala desde la cual comienza el procedimiento, otro caso fue computado usando un espectro que incluye la parte de gran escala:

$$
\begin{aligned}
E(k)=C \varepsilon^{2 / 3} k^{-5 / 3}[ & \left.\frac{k L}{\left((k L)^{2}+c_{L}\right)^{1 / 2}}\right]^{5 / 3+p_{0}} \\
& \times \exp \left(-\beta\left[(k \eta)^{4}+c_{\eta}^{4}\right]^{1 / 4}-c_{\eta}\right)
\end{aligned}
$$

donde $p_{0}=4$ y $c_{L}$ se determina de modo que la integral de $E(k)$ se reduzca a la energía total $3 / 2 u_{\text {rms }}^{2}$. Para este caso $\operatorname{Re}_{\tau}=407$ y la secuencia es $\left(N_{1}^{3}=8^{3} ; M=8\right)$. Los resultados aparecen también en la Figura 2. Como es de esperar, el rango de ley de potencia se hace más estrecho, debido a la reducción del rango inercial, pero todavía los exponentes de escala son próximos a los $\mathrm{K} 41$. El parámetro $t_{n}$ es la variable que determina de forma más importante el grado de distorsión introducida a cada escala. En la forma de la ecuación (10), $t_{n}$ es equivalente al tiempo promedio que le toma a una partícula moverse entre nodos adyacentes. Esto permite suficiente distorsión para dar origen a propiedades turbulentas no-gaussianas, evitando a la vez la rerandomización provocada por la combinación de velocidades no correlacionadas [1]. Esta selección de $t_{n}$ depende no obstante de una longitud arbitraria $\left(\ell_{n}\right)$, no relacionada con la dinámica intrínseca de la turbulencia. Esto motiva a explorar una nueva formulación que utilice un parámetro basado en la física del fenómeno.

\section{MÉTODO MTLM}

El tiempo característico a una escala $r$ (llamado "turnover time") en un flujo turbulento se puede estimar como

$$
t_{n} \sim \frac{r}{\delta u_{r}}
$$

siendo $\delta u_{r}$ un valor típico para las velocidades relativas asociadas con dicha escala. Es bien sabido que en la fenomenología de turbulencia se considera que el flujo medio de energía a través de la escala $r$ en el rango inercial es constante e igual a la tasa de disipación $\varepsilon$ (la así llamada "cascada" de Richardson). Esta disipación es determinada por los movimientos de gran escala comparables a la escala integral de la turbulencia, $L$, y se estima como $L \sim u_{\mathrm{rms}}^{3} / \varepsilon$ ). Por lo tanto,

$$
\varepsilon \sim \frac{u_{\mathrm{rms}}^{3}}{L} \sim \frac{\left(\delta u_{r}\right)^{3}}{r}
$$

de modo que la velocidad sigue una ley de escala según $\delta u_{r} \sim(r \varepsilon)^{1 / 3}$. Tomado como escala $r$ la separación $\ell_{n}$ entre puntos adyacentes del $n$-ésimo nivel, tenemos que

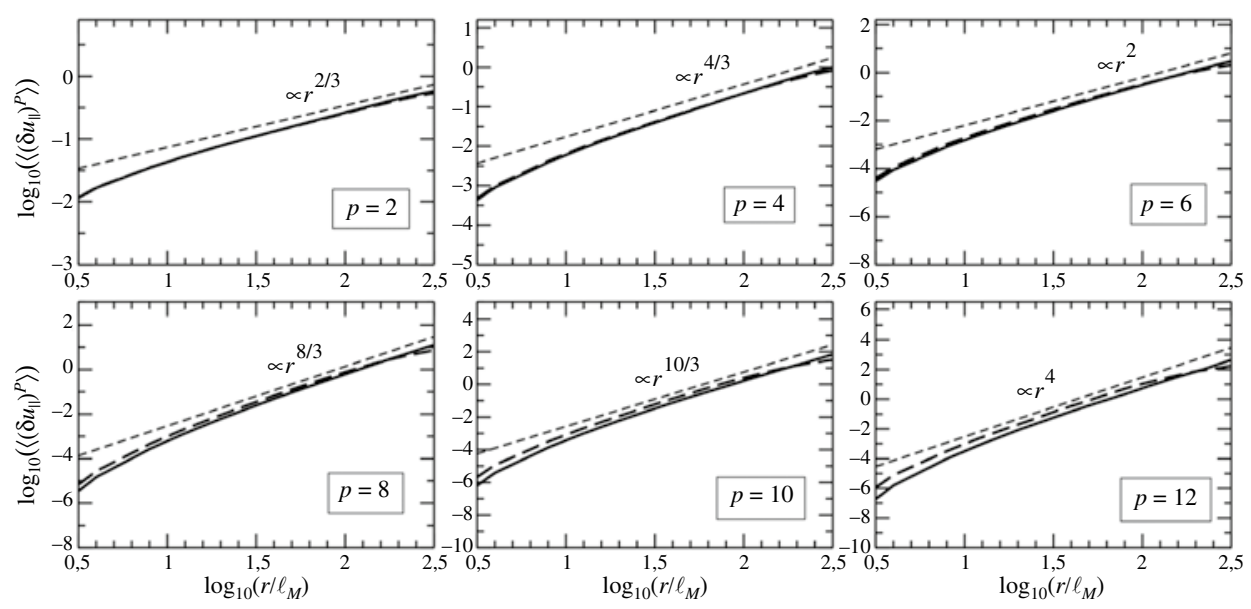

Figura 2. Funciones de estructura de velocidad para un campo MMLM de $1.024^{3}$ puntos. Líneas llenas: usando el espectro (15); líneas segmentadas: usando el espectro (16). 


$$
\tau_{n} \sim \ell_{n}^{2 / 3} / \varepsilon^{1 / 3}
$$

Por otra parte, ya que se utiliza un filtro pasabajos, la velocidad r.m.s. $\left(u_{\mathrm{rms}, n}\right)$ que interviene en el cálculo de $t_{n}$ es aproximadamente igual a la velocidad r.m.s. $\left(u_{\text {rms }}\right)$ del flujo completo, en el caso de un espectro típico donde la energía está contenida principalmente en las grandes escalas. Ya que $u_{\text {rms }} \sim(\varepsilon L)^{1 / 3}$, la razón entre $t_{n}$ y el tiempo turnover se puede estimar como

$$
\frac{t_{n}}{\tau_{n}} \approx\left(\frac{\ell_{n}}{L}\right)^{1 / 3}
$$

$\tau_{n}$ es considerado como representativo del tiempo que le toma a una estructura de tamaño $r$ experimentar una distorsión considerable por acción de movimientos turbulentos, y transferir su energía a escalas todavía menores por medio de la cascada. La relación (20) muestra que $t_{n}$ es progresivamente menor que $\tau_{n}$ a medida que se avanza de un nivel al siguiente, de modo que existe una creciente disparidad entre $t_{n}$ y el tiempo característico de la turbulencia real para aquella escala en particular en la cual el mapeo está siendo aplicado. Por consiguiente, el procedimiento será modificado de modo que las partículas se desplacen por un tiempo equivalente acumulado $\tau_{n}$ dado por la ecuación (19) a cada nivel, en lugar de $t_{n}$.

Fue establecido en [1] que los desplazamientos de las partículas no deben diferir mucho de su separación original $\ell_{n}$. De esa forma, regiones de intensos gradientes de velocidad son generadas principalmente por partículas de trayectorias convergentes cuya separación inicial era del orden de $\ell_{n}$, y que tenían por lo tanto velocidades correlacionadas al inicio del desplazamiento. Para desplazamientos mayores, la descorrelación entre las velocidades de las partículas que arriban a la vecindad de un punto conduce a una rerandomización del campo de velocidad, destruyendo la coherencia de fases introducida en el nivel precedente. Debido a esto, el avance total se efectuará en varios pasos a cada nivel, en forma tal que el desplazamiento r.m.s. en cada paso sea aún del orden de $\ell_{n}$. Más precisamente, para cada nivel en la jerarquía de escalas, la secuencia de mapeo (9), interpolación y proyección es aplicada $D$ veces, con

$$
D=\left[\frac{u_{\mathrm{rms}, n}}{\left(\ell_{n} \varepsilon\right)^{1 / 3}}\right]
$$

([·] denota entero más próximo), y cada $t_{n}$ a ser usado en (9) está dado por su correspondiente fracción de $\tau_{n}$, es decir,

$$
t_{n}=\frac{1}{D}\left(\frac{\ell_{n}^{2}}{\varepsilon}\right)^{1 / 3}
$$

Se puede así acumular un tiempo total $\tau_{n}$ mediante esta secuencia de $D$ pasos, cada uno con duración $t_{n}$. Después de cada uno de estos desplazamientos la interpolación para obtener $\mathbf{v}_{n}^{<}$en los puntos eulerianos $\mathbf{x}_{n}$ es aplicada. Estas velocidades son reescaladas directamente en el espacio físico, para mantener constante la velocidad r.m.s. del campo, y luego se aplica la proyección sobre la parte solenoidal. Para abreviar, este procedimiento modificado se designará como MTLM (Multiscale Turnover Lagrangian Map).

Un nuevo campo sintético de $1.024^{3}$ puntos fue generado con el método MTLM. El espectro de energía y las otras condiciones son las mismas que las usadas en el último caso MMLM mostrado. Los resultados para las funciones de estructura de velocidad en este campo se presentan en la Figura 3. Las dos líneas segmentadas finas en cada gráfico indican leyes de potencia de $r$ de acuerdo con los exponentes del escalamiento de Kolmogorov $\zeta_{p}=p \beta$. Los escalamientos según los exponentes generalmente reportados en la literatura de flujos turbulentos [4-8] son también mostrados (para $p=2$ y $p=4$ los valores de Kolmogorov no se muestran). Se puede observar que el campo MTLM presenta una concordancia mucho mejor con el comportamiento intermitente conocido en turbulencia que los casos previos computados en la sección anterior. Existe una desviación clara y sistemática con respecto a los exponentes de Kolmogorov, y las pendientes de las funciones de estructura están notablemente próximas a los valores asociados con turbulencia real. Los exponentes de referencia usados para comparación en la Figura 3 son un promedio de las mediciones experimentales de [5] para $\operatorname{Re}_{\lambda}=515$, 536 y 852 . El promedio de los exponentes obtenidos con simulación numérica directa (DNS) por Gotoh [8] a $\operatorname{Re}_{\lambda}=381$ y 460 difieren de los valores usados 
para comparación en un rango de $0,4 \%$ a $1,7 \%$ (para $p \leq 10$ ).

Las leves oscilaciones que se observan para los órdenes más altos calculados se pueden atribuir a la naturaleza aproximada del método, y suponer asociadas a la secuencia discreta de octavas durante la construcción del campo de velocidades. Desde una perspectiva de más conjetura, estas oscilaciones podrían ser incluso compatibles con cierto comportamiento ondulante observado a veces en funciones de estructura de alto orden (ver [4]). La hipotética existencia de oscilaciones log-periódicas en las exponentes de escala [9-10] podría surgir de una razón preferencial de fragmentación en la cascada desde los vórtices mayores hacia los más pequeños y significar una invariancia de escala de tipo discreto.

Aplicando el método MTLM es posible observar ahora intermitencia de rango inercial también en un caso más pequeño, con $512^{3}$ puntos (y $\operatorname{Re}_{\lambda}=253$ ). Las correspondientes funciones de estructura aparecen también en la Figura 3. Para las funciones de orden $p=8,10$ y 12 , la buena concordancia cubre una década de escalas. A pequeñas escalas, las funciones se separan de la tendencia como ley de potencia a $\log _{10}\left(r / \ell_{M}\right) \approx 0,7$. Esta longitud está asociada aproximadamente con el número de onda $k \approx 2 \pi$ $/ r=204$, siendo esto consistente con el número de onda al cual tiene lugar la separación del espectro de energía del comportamiento de rango inercial en el extremo de altos números de onda. Una regresión lineal ajustada a las funciones de estructura (para el caso $1.024^{3}$ ) en el rango $0,7 \leq \log _{10}\left(r / \ell_{M}\right) \leq 2$ da, para los órdenes $p=8,10$ y 12 los exponentes $\zeta_{8}=2.235$, $\zeta_{10}=2.504$ y $\zeta_{12}=2.703$ respectivamente, en muy buena concordancia con los valores experimentales y numéricos obtenidos en turbulencia de NavierStokes.

\section{ANÁLISIS MULTIFRACTAL DE LA DISIPACIÓN}

La visión convencional de la transferencia de energía entre escalas como un proceso en cascada desde los vórtices mayores hacia los más pequeños hasta alcanzar las escalas disipativas, ha conducido a la idea de que las características de este proceso podrían manifestarse en el producto final de la cascada, vale decir, en el campo de distribución espacial de la disipación de energía. Para evitar confusión con la disipación media $\varepsilon$, la disipación local en la posición $\mathbf{x}$ se designará como $\tilde{\varepsilon}(\mathbf{x})$. Esta variable es determinada por el tensor de tasa de deformación $S_{i j}=1 / 2\left(\partial_{i} u_{j}+\partial_{j} u_{i}\right)$ según la relación

$$
\tilde{\varepsilon}(\mathbf{x})=2 \vee S_{i j}(\mathbf{x}) S_{i j}(\mathbf{x})
$$

Como ejemplo, la Figura 4 muestra perfiles unidimensionales de la disipación local, a lo largo de la coordenada $x$ del dominio computacional,
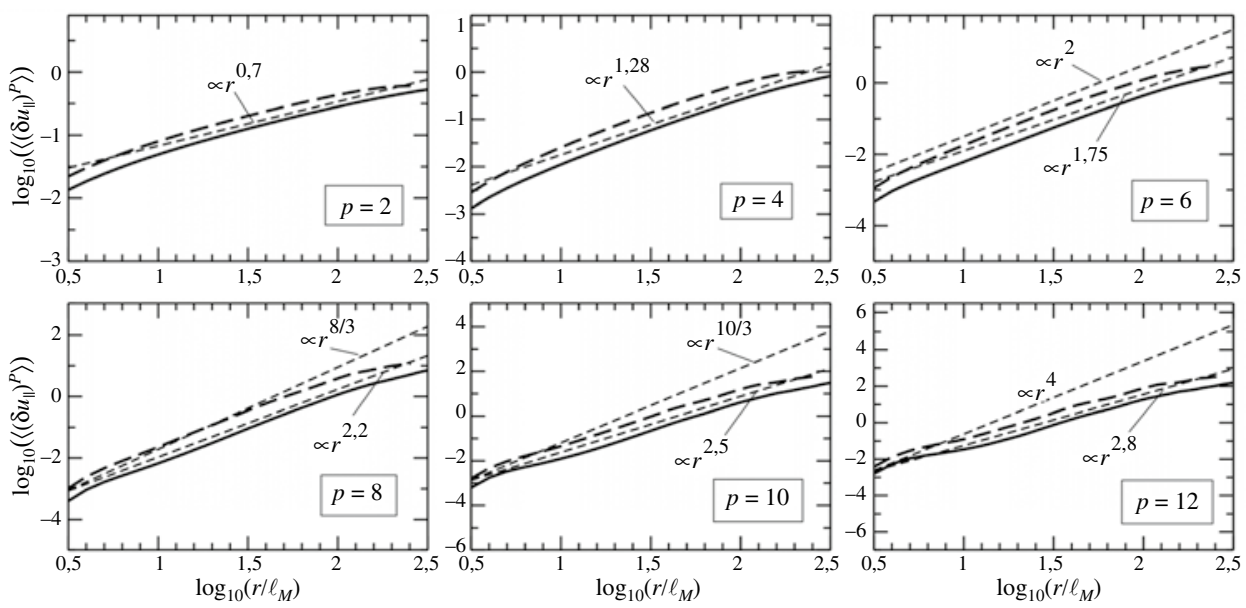

Figura 3. Funciones de estructura de velocidad para un campo MTLM de $1.024^{3}$ (líneas llenas) y $512^{3}$ puntos (líneas segmentadas). Líneas segmentadas finas indican leyes de potencia con los exponentes que se indican. 
para tres posiciones $y$, a través de un plano de $z$ constante, obtenidos del campo de velocidades MTLM con $1.024^{3}$ nodos. Se puede observar una distribución espacial de la disipación extremadamente intermitente, con puntos dispersos, en donde la disipación alcanza valores particularmente elevados, excediendo el valor medio por más de un orden de magnitud, tal como se ha observado en campos turbulentos reales.

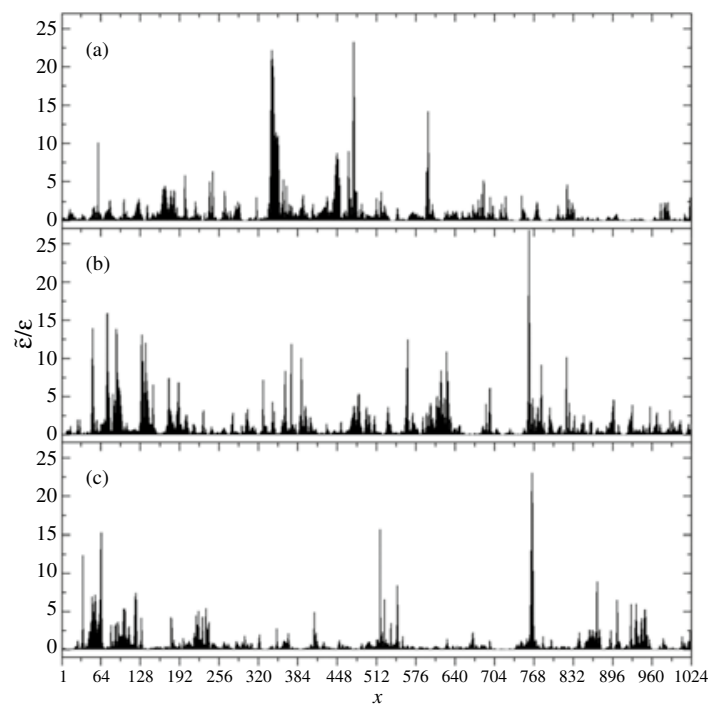

Figura 4. Disipación local de energía normalizada por la disipación media, para (a) $y=256$, (b) $y=512$ y (c) $y=768$, en un plano de z constante. Las coordenadas $(x, y)$ están en unidades de la malla computacional.

Una cascada (o un proceso multiplicativo) se puede describir usualmente mediante el formalismo multifractal, el cual es particularmente apto para describir este tipo de medidas fuertemente intermitentes [9]. Una medida multifractal es esencialmente aquella para la cual las dimensiones Renyi $D_{q}$ varían con $q$, de modo que se requiere un espectro de dimensiones $D_{q}$ para caracterizarla (en contraste con un monofractal para el cual $D_{q}=D_{0}$ cuando $q>0$, siendo así descrito por su dimensión de capacidad, $D_{0}$, y la medida es uniforme sobre su soporte). Se cree que la disipación en turbulencia puede describirse, con buena aproximación, como una de tales medidas multifractales [10], con conjuntos de diferentes dimensiones fractales topológicamente entrelazados.
Por lo tanto, la medida específica a considerar es $\tilde{\varepsilon}(\mathbf{x})$. La disipación acumulada $E_{r}$ en una región $\Omega$ de tamaño característico $r$ es

$$
E_{r}=\iiint_{\mathbf{x}^{\prime} \in \Omega(\mathbf{x})} \tilde{\varepsilon}\left(\mathbf{x}^{\prime}\right) \mathrm{d}^{3} \mathbf{x}^{\prime}
$$

y la disipación total en el dominio completo, de tamaño $H$, se designará como $E_{t}$. Específicamente, las regiones $\Omega$ se tomarán como cubos de lado $r$ centrados en las posiciones $\mathbf{x}$.

La caracterización de un multifractal se puede hacer en términos de las dimensiones $D_{q}$ o por medio de su espectro de singularidades. Después de cubrir el volumen con cubos iguales de tamaño $r$, se puede postular una relación local de escalas de la forma

$$
\frac{E_{r}}{E_{t}} \sim\left(\frac{r}{H}\right)^{\alpha}
$$

y el exponente Hölder $\alpha$ cuantifica la intensidad de la singularidad en $\mathbf{x}$ cuando $r \rightarrow 0$. Para un multifractal, $\alpha$ depende de la posición $\mathbf{x}$, y $E_{r}$ sigue así una ley de escala con una multitud de exponentes sobre diferentes conjuntos, a cada uno de los cuales le corresponde una dimensión Hausdorff que se designará como $f(\alpha)$. La función $f(\alpha)$ es el espectro de singularidades. Ya que una distribución puede describirse también por sus momentos, una relación similar a (25) es escrita para la suma de $E_{r}^{q}$ sobre todos los $\Omega$-cubos disjuntos de tamaño $r$ :

$$
\sum_{\Omega}\left(\frac{E_{r}}{E_{t}}\right)^{q} \sim\left(\frac{r}{H}\right)^{\gamma(q)}
$$

Dado que $f(\alpha)$ es una dimensión Hausdorff para el conjunto con exponente $\alpha$, el número de cubos $N(\alpha)$ en donde $E_{r}$ tiene una singularidad de intensidad comprendida entre $\alpha$ y $\alpha+\mathrm{d} \alpha$ se puede estimar como

$$
N(\alpha) \mathrm{d} \alpha \sim g(\alpha)\left(\frac{r}{H}\right)^{-f(\alpha)} \mathrm{d} \alpha
$$

para algún prefactor $g(\alpha)$, que no es relevante. Esto se basa en la definición de dimensión fractal.

La suma en la ecuación (26) puede evaluarse integrando sobre todos los valores de $\alpha$. Empleando las ecuaciones (25) y (27) dicha integración produce 


$$
\begin{gathered}
\sum_{\Omega}\left(\frac{E_{r}}{E_{t}}\right)^{q} \sim \int\left(\frac{r}{H}\right)^{\alpha q} g(\alpha)\left(\frac{r}{H}\right)^{-f(\alpha)} \mathrm{d} \alpha \\
=\int \exp ([f(\alpha)-q \alpha] \ln (H / r)) g(\alpha) \mathrm{d} \alpha \\
\sim \exp ([f(\alpha(q))-q \alpha(q)] \ln (H / r))
\end{gathered}
$$

en donde la integral ha sido aproximada por su valor asintótico para $r \rightarrow 0$ según el método de Laplace. Por consiguiente, $\alpha(q)$ debe ser el valor de $\alpha$ para el cual

$$
\left.\frac{\mathrm{d} f}{\mathrm{~d} \alpha}\right|_{\alpha(q)}-q=0
$$

y $f^{\prime \prime}(\alpha(q))<0$. De la última relación en (28) se puede observar que

$$
\sum_{\Omega}\left(\frac{E_{r}}{E_{t}}\right)^{q} \sim\left(\frac{r}{H}\right)^{-[f(\alpha(q))-q \alpha(q)]}
$$

y comparando este resultado con la ecuación (26) se concluye que

$$
f(\alpha(q))=q \alpha(q)-\gamma(q)
$$

Derivando esta última ecuación con respecto a $q$, y haciendo uso del resultado (29) se tiene que

$$
\alpha(q)=\frac{\mathrm{d} \gamma}{\mathrm{d} q}
$$

Las ecuaciones (31) y (32) constituyen por lo tanto una transformación de Legendre de la función $\gamma$ $(q)$.

La computación de las sumas $\Sigma_{\Omega}\left(E_{r} / E_{t}\right)^{q}$ se efectuó para 43 valores de $q$ en el intervalo $q \in[-2,3,25]$, sobre todos cubos disjuntos posibles de tamaño $9,3 \leq r / \eta \leq 238$ en el campo MTLM de $1.024^{3}$ puntos. Este intervalo de escalas permite abarcar una parte substancial de los rangos inercial y disipativo. Para valores mayores de $r$ el número de cubos resulta demasiado reducido para muestreo estadístico significativo. Resultados de $\Sigma_{\Omega}\left(E_{r} / E_{t}\right)$ ${ }^{q}$ para valores representativos de $q$ se muestran en la Figura 5. Una ley de potencia consistente con la ecuación (26) se satisface bien en general, tornándose un poco más ambigua, pero aún razonable, para los exponentes más altos. Se utilizó regresión de mínimos cuadrados para obtener los exponentes $\gamma$ $(q)$ de los momentos a partir de estos datos. Para este fin, la regresión se debe restringir a un rango de $r$, para el cual el amortiguamiento viscoso introducido por el espectro de energía impuesto no afecte los resultados. Es sabido que este límite inferior se sitúa
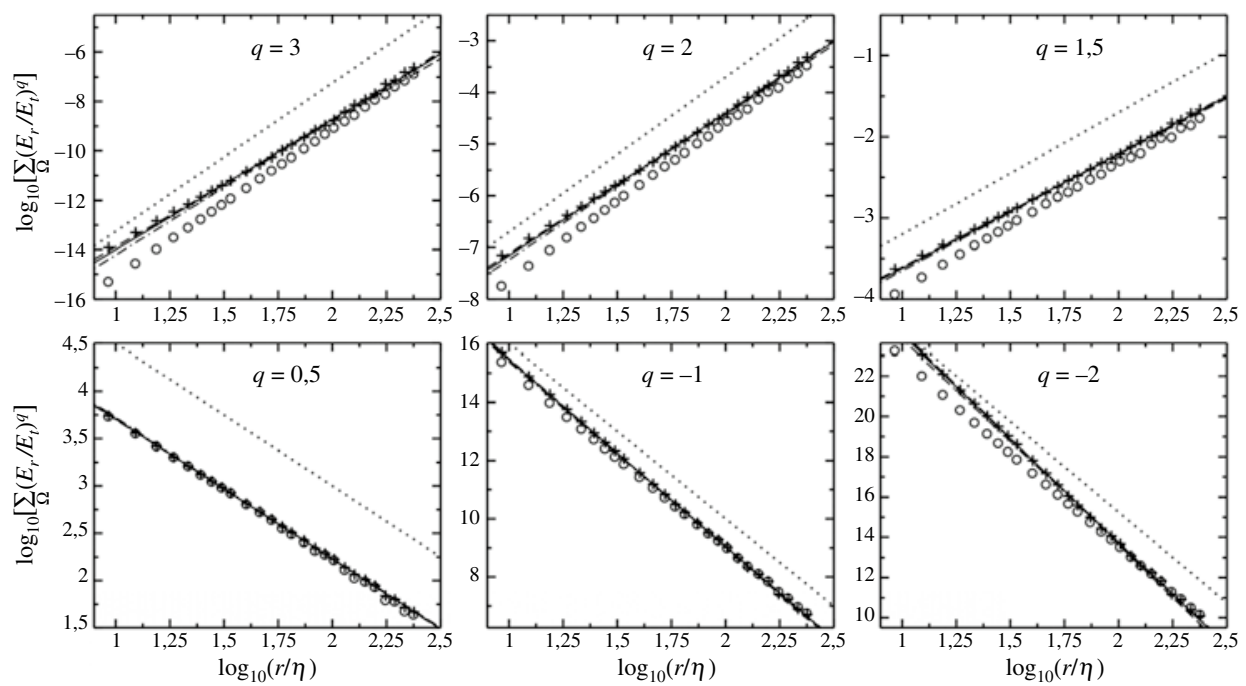

Figura 5. Momentos de la disipación acumulativa normalizada, para los órdenes $q$ indicados, como función del tamaño $r$ de los $\Omega$-cubos. (+): campo MTLM 1.02433; (o): campo MMLM 1.0243; línea punteada: escalamiento K41; línea llena: regresión sobre rango A; línea segmentada: regresión sobre rango B; línea segmentada-punteada: regresión sobre rango C. 
alrededor de $r / \eta>20$ en turbulencia real. Por otra parte, para remover efectos provenientes desde el rango de grandes escalas, también se excluyen las escalas correspondientes a $r>0,2 L$ (con $L$ la escala integral). Esto determina un rango de $0,81<\log _{10}(r /$ $\left.\ell_{M}\right)<1,81$, el cual es referido como "rango A" en lo que sigue (ya que $\ell_{M}=3,09 \eta$, este rango equivale a $20<r / \eta<200)$. Con el fin de apreciar la variabilidad de estos resultados debido a la incerteza en el rango apropiado de escalamiento, se toman dos rangos adicionales, los cuales modifican por un factor 2 el límite inferior o superior de $r / \eta$ en el rango A: rango $\mathrm{B}$ para $0,81<\log _{10}\left(r / \ell_{M}\right)<1,51 \mathrm{y}$ rango $\mathrm{C}$ para $1,11<\log _{10}\left(r / \ell_{M}\right)<1,81$. En la Figura 5 se presentan las regresiones sobre estos tres rangos de escalas.

La Figura 6 muestra los exponentes $\gamma(q)$ obtenidos usando el rango A (símbolos + ). Nótese que en el caso de disipación no intermitente y que llena todo el espacio, como implicaría la teoría K41, las dimensiones $D_{q}$ serían iguales a 3 para todo $q$, y, por lo tanto, $\gamma(q)$ estaría dado por la función lineal $\gamma(q)=3(q-1)$ [ver la ecuación (33)]. Para comparación, la Figura muestra también los exponentes obtenidos para un campo generado con el procedimiento MMLM para las mismas condiciones. Es interesante, y consistente con los resultados del escalamiento de las funciones

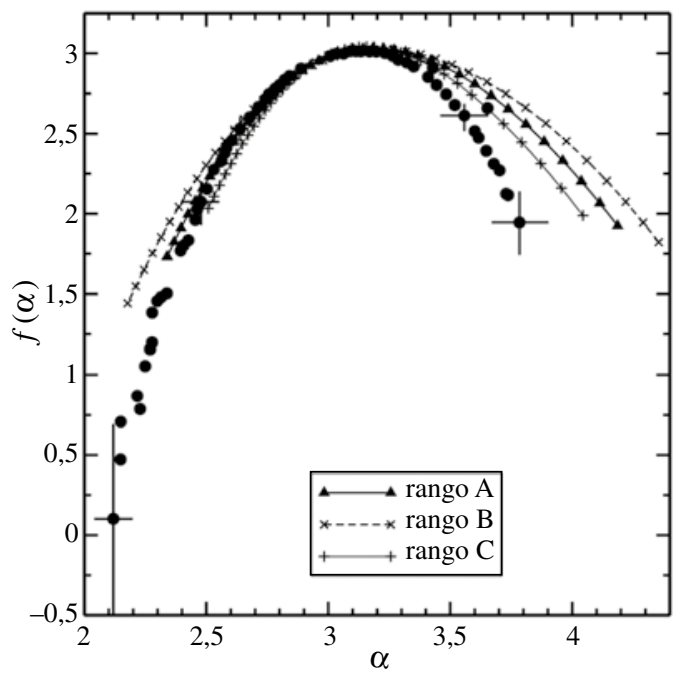

Figura 6. Espectro de singularidades para el campo MTLM de $1.024^{3}$ nodos. Los símbolos circulares corresponden a datos experimentales de [13]. de estructura visto anteriormente, que el MMLM produce esencialmente un escalamiento del tipo K41 en el rango de escalas disipativas.

El espectro de singularidades $f(\alpha)$ obtenido a partir de los exponentes $\gamma(q)$ se muestra en la Figura 6, junto con datos tomados de [13], provenientes de observaciones experimentales en flujos de laboratorio y atmosféricos. El espectro del campo sintético se asemeja razonablemente bien al comportamiento observado en turbulencia real, y hay buena concordancia cuantitativa en la región del núcleo $2,5<\alpha<3,5$ del espectro. Para el extremo derecho del espectro, el $f(\alpha)$ sintético no decae tan rápido como en el caso experimental, mientras que la concordancia es mejor sobre el extremo izquierdo.

Hay que recordar que elevados $\alpha$ corresponden a momentos de elevados órdenes $q$ negativos, mientras que $\alpha$ reducidos están asociados con los más altos $q$ positivos. Por consiguiente, además del hecho obvio de que los extremos del espectro son probablemente más afectados por la incerteza en el rango apropiado de escalamiento, se debe considerar también que momentos de orden negativo enfatizan los valores más pequeños (cercanos a cero) de la disipación en el campo. Estas zonas de baja disipación surgen principalmente en las regiones en donde el mapeo lagrangiano produce una escasez relativa de partículas fluidas (una rarefacción en la densidad numérica local de las mismas), de modo que la interpolación produce un gradiente local de velocidad reducido (en forma complementaria a las regiones en donde las partículas convergen, originando elevados gradientes de velocidad y alta disipación). Debido a las características de la construcción del campo de velocidades, la estructura de aquellas regiones con distribuciones de velocidad suavizadas e intensidad de turbulencia deprimida es definida en su mayor parte durante las primeras etapas del procedimiento de síntesis (es decir, por las deformaciones introducidas en las mayores escalas de la secuencia), mientras que las zonas de alta disipación, enfatizadas por $q>0$, son resultado de la acumulación de distorsión sobre distorsión del campo local de velocidad. En este sentido, el procedimiento actúa más eficazmente en la construcción de las regiones más singulares que en el desarrollo de las más regulares. Esto puede explicar por qué la concordancia sobre el 
lado izquierdo del espectro de singularidad es mejor que sobre el lado derecho.

Tomando el logaritmo de la ecuación (26) se puede ver que $\chi(q)$ está relacionada con las dimensiones Renyi mediante

$$
D_{q}=\frac{\gamma(q)}{q-1}=\frac{q \alpha(q)-f(\alpha(q))}{q-1}
$$

Además, se puede notar que la relación (25) implica que la disipación promediada sobre los cubos de tamaño $r$ sigue una ley de escala del tipo

$$
\frac{\varepsilon_{r}}{\varepsilon} \sim\left(\frac{r}{H}\right)^{\alpha-3}
$$

y entonces, un comportamiento singular con $r \rightarrow 0$ ocurre para $\alpha<3$, mientras que en puntos con $\alpha>3$ la disipación es regular. Por supuesto, en la situación física real los gradientes de velocidad son suavizados por la viscosidad a escalas suficientemente pequeñas, y singularidades en el sentido matemático no ocurren, estando este comportamiento cuasisingular reflejado por valores locales de disipación de extrema intensidad, como los observados en la Figura 4.

La buena correspondencia en el núcleo del espectro sugiere que algunas propiedades importantes de la naturaleza multifractal de la disipación turbulenta son bien reproducidas por la aproximación MTLM. En lo que sigue se toma el rango A como línea base para comparación, y los resultados para los rangos $\mathrm{B}$ y $\mathrm{C}$ se indican entre paréntesis, en dicho orden. De acuerdo con la ecuación (29) el valor máximo para $f(\alpha)$ ocurre para $q=0$ y, por lo tanto, por la ecuación (33) se tiene

$$
D_{0}=f(\alpha)_{\max }=3
$$

que es la dimensión del espacio que soporta a la medida (en este caso, la disipación local). El valor de $f(\alpha)_{\max }$ ocurre para $q=3,18(3,21,3,16)$ en el campo sintético, lo cual se sitúa próximo al valor $q \approx 3,13$ encontrado en el espectro de singularidades turbulento [13]. Más importante es la dimensión $D_{1}$ (la denominada dimensión de información), que corresponde a la dimensión del conjunto que contiene asintóticamente a la disipación. Si uno considera subconjuntos del soporte que tengan una fracción $\chi$ de la medida, $D_{1}$ es la dimensión del subconjunto que tiene la mínima dimensión $D_{0}$ cuando $\chi \rightarrow 1$. De acuerdo a su definición en la ecuación (26), se tiene que $\gamma(1)=0$, de manera que para $q=1$ tenemos

$$
f(\alpha(1))=\alpha(1)
$$

y por (33), también se cumple que $D_{1}=\alpha(1)$. El valor de $D_{1}$ derivado en esta forma desde el espectro del campo sintético es $D_{1}=2,89(2,85,2,93)$, el cual concuerda bien con el valor $D_{1} \approx 2,87$ encontrado en turbulencia real [13].

La integración en la ecuación (28) da la dimensión fractal (dimensión "box-counting") para el caso cuando $q=0$. Tomando solo las regiones en donde la disipación tiene un comportamiento singular se restringe el intervalo de integración a $\alpha<3$. Ya que $f(\alpha)$ es monótonamente creciente en dicho intervalo, el máximo valor de $f(\alpha)$ en tal intervalo restringido ocurre para $\alpha \rightarrow 3$. Este valor máximo domina el valor asintótico de la integral en estas condiciones, y por lo tanto la dimensión Hausdorff del conjunto de singularidades es $f(3)$. Para los datos de turbulencia real, el valor obtenido en [13] es $f(3) \approx 2,96$, mientras que para el campo sintético se obtiene $f(3) \approx 2,97$, en excelente concordancia.

También es posible calcular el exponente de intermitencia $\mu$, definido tradicionalmente como

$$
\frac{\left\langle\varepsilon_{r}^{2}\right\rangle}{\varepsilon^{2}} \sim\left(\frac{L}{r}\right)^{\mu}, \text { para } \eta<r<<L
$$

Para momentos de bajo orden la aproximación lognormal es válida, lo cual es equivalente a una forma cuadrática para $\gamma(q)$, obteniéndose [13]

$$
\mu=\left.\frac{d^{2} \gamma(q)}{d q^{2}}\right|_{q=0} .
$$

El resultado obtenido de las curvas de la Figura 7 (computado numéricamente con interpolación de orden 10) es $\mu=0,33(0,41,0,25)$. Esto es algo mayor, pero aun dentro del rango de los valores experimentales a menudo citados en turbulencia real, los que se ubican en el rango $\mu=0,25 \pm 0,05$ [14]. Se debe considerar además que este parámetro está asociado con una medida altamente intermitente, 
para la cual la convergencia de la estadística resulta más difícil. Para otra realización de un campo de flujo sintético MTLM de $1.024^{3}$ puntos, el valor obtenido fue $\mu=0,24$ [16].

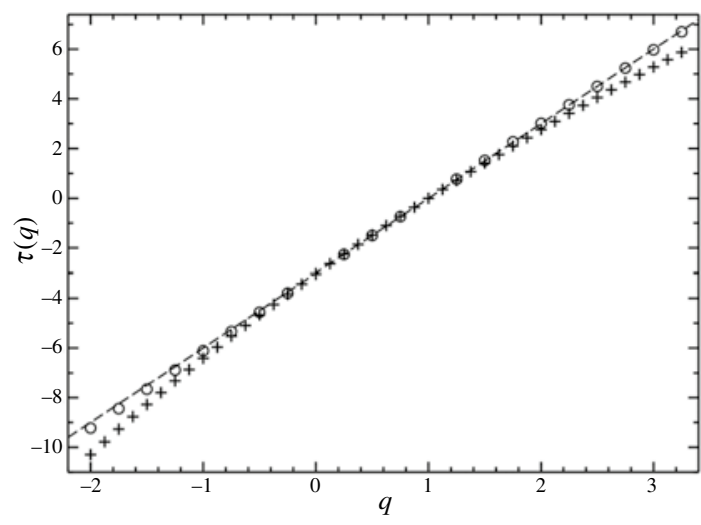

Figura 7. Exponentes de momentos como función de $q .(+)$ : campo MTLM 1024; ; (o): campo MMLM $1024^{3}$; línea segmentada: escalamiento K41.

En resumen, se encuentra que los campos sintéticos MTLM exhiben multifractalidad en el campo de disipación implícito, con niveles de intermitencia que son comparables con aquellos de la turbulencia real. Por contraposición, si un parámetro de mapeo no conmensurable con la escala de tiempo fenomenológica de Kolmogorov se utiliza para las distorsiones de cada escala espacial, el campo de disipación permanece esencialmente similar al de la teoría K41, sin escalamiento intermitente. Luego, la evidencia es que para el desarrollo de la intermitencia es imperativo que se permita a las pequeñas escalas evolucionar al menos durante un tiempo igual a su escala de tiempo característico (el denominado "turnover time").

\section{CONCLUSIONES}

En este trabajo se aborda en primer lugar el problema de la intermitencia y el escalamiento anómalo en los campos turbulentos sintéticos MMLM. Adicionalmente a lo aquí presentado, se han estudiado también las propiedades estadísticas y de estructura espacial de los campos de vorticidad y deformación y el campo de presión inducido, el cual está implícito en el campo de velocidades obtenido que ha sido analizado (tales resultados se presentan en [15]). En todos estos casos, lo observado en el campo sintético MTLM está en notable concordancia con sus contrapartes en turbulencia real. El principal hallazgo aquí es que con un parámetro de mapeo según la ecuación (8) no aparece escalamiento anómalo significativo en el rango inercial. Si bien dicho parámetro es suficiente para generar los momentos estadísticos de bajo orden que determinan muchas de las características nogaussianas de la turbulencia, la deformación inducida en el campo de velocidad es progresivamente más débil a medida que la escala de longitudes se reduce. Con la forma (8), el parámetro de tiempo no está relacionado con la dinámica intrínseca de la cascada turbulenta. Un procedimiento modificado ha sido explorado para el cual el parámetro es calculado de un modo tal que la distorsión total acumulada tiene lugar, en promedio, durante el tiempo "turnover" lagrangiano local, el cual es particular para cada escala. Se encuentra que esta segunda forma es capaz de reproducir adecuadamente el fenómeno de escalamiento anómalo.

En relación con la estructura espacial de la disipación de energía, ésta es examinada mediante análisis multifractal y su espectro de singularidades es determinado. Los resultados concuerdan razonablemente bien con las características multifractales conocidas de la disipación en turbulencia real. De modo consistente con el análisis de las funciones de estructura, el campo de disipación generado con la aproximación MMLM no produce escalamiento multifractal.

Dos de los componentes fundamentales de la turbulencia son la autodistorsión del campo de velocidad y la estructura multiescala de dicho campo. El primero de éstos es proporcionado en nuestro caso sintético por el mapeo lagrangiano, mientras que el segundo es explícitamente introducido por la jerarquía de escalas usada. La construcción del campo de velocidad es esencialmente cinemática, sin ningún efecto dinámico continuo para producir escalas más pequeñas a partir de las escalas menores, sino que en lugar de ello las escalas menores (con estadística gaussiana) son directamente introducidas a cada nivel de la secuencia, y luego se les permite que se deformen por sí mismas y por la acción de las escalas mayores que las precedieron. Estas escalas pequeñas no son directamente un producto de las escalas mayores, sino que ellas (junto con su estadística gaussiana inicial) son agregadas 
arbitrariamente sobre el campo de velocidad de escala mayor. Lo que surge directamente del campo de escala mayor es la distribución espacial de las localizaciones en donde estas fluctuaciones adicionales son más fuertemente distorsionadas. La deformación acumulativa secuencial de estos campos de velocidad en el proceso sintetizador juega el rol conectivo entre escalas. En este caso sintético ninguna transferencia dinámica de energía (cascada de energía) tiene lugar, ya que la cantidad apropiada de energía a cada nivel es impuesta por construcción durante el reescalamiento a través del espectro de energía. En su lugar se tiene una especie de "cascada de estructura espacial", por la cual se desarrolla intermitencia espacial a partir de las inhomogeneidades de la deformación que ha ocurrido en los campos de velocidad de mayor escala.

Para explorar este punto, se efectuaron pruebas adicionales en las cuales esta acumulación de intermitencia espacial fue suprimida artificialmente. Esto se hizo dejando que los campos de velocidad a cada escala fueran deformados como antes y aplicando el mismo parámetro de mapeo, pero usando para cada escala el campo de velocidad inicial gaussiano. En esta forma, los efectos de la distorsión a cada una de las escalas no son propagados acumulativamente en la secuencia, y sólo son combinados en la etapa final. En este caso, el campo final de velocidad permaneció básicamente gaussiano, y no se observó escalamiento anómalo alguno.

Los resultados indican que adicionalmente a la autodistorsión del campo de velocidad y a la deformación secuencial de una multiplicidad de escalas, la cantidad apropiada de deformación a cada escala es crucial para la aparición del escalamiento anómalo y la manifestación de la real intermitencia de las escalas disipativas. Los dos primeros elementos son suficientes para producir gradientes de velocidad concentrados, esencialmente correctos momentos estadísticos cúbicos dependientes de gradientes de velocidad, y la interrelación entre vorticidad y tasa de deformación, pero el escalamiento anómalo y la intermitencia sólo surgen cuando se introduce la información física apropiada sobre la magnitud de la distorsión a cada escala. Esta información, en la forma del tiempo turnover (K41) particular para cada escala, se torna así en el único suministro de dinámica real de turbulencia (además del espectro de energía) que es provisto. Nótese que la escala de tiempo prescrita sigue el escalamiento estándar K41 y no incluye a priori correcciones de intermitencia prescritas. Las correcciones de intermitencia al escalamiento de las funciones de estructura surgen del procedimiento mismo.

Además de contribuir a una mejor comprensión de los procesos cruciales que son necesarios para la manifestación de la intermitencia en campos turbulentos, el hecho de que el MTLM logre reproducir adecuadamente esta característica con bajo costo computacional, lo convierte en una valiosa herramienta para la generación de condiciones iniciales realistas o condiciones de contorno en simulación numérica de flujos turbulentos. Para esto último se explorará a futuro la posibilidad de generar campos de velocidad anisotrópicos, mediante la distorsión individual de las componentes de velocidad para introducir esfuerzos de Reynolds prescritos. De igual modo, la advección de un campo escalar pasivo por acción de campos de velocidad sintéticos generados con este método ha mostrado que las peculiares características de la turbulencia escalar, la cual presenta una intermitencia todavía más intensa, son también correctamente reproducidas por el método [16].

\section{AGRADECIMIENTOS}

El autor agradece el apoyo brindado por CONICYT, a través del Proyecto FODECYT 11080025.

\section{REFERENCIAS}

[1] C. Rosales and C. Meneveau. "A minimal multiscale Lagrangian map approach to synthesize non-Gaussian turbulent vector fields". Physics of Fluids. Vol. 18, pp. 075104:114. 2006.

[2] A. Monin and A. Yaglom. "Statistical Fluid Mechanics: Mechanics of Turbulence". MITPress. Vol. 2. Cambridge, Massachusetts, USA. 1975.

[3] K.R. Sreenivasan and R.A. Antonia. "The phenomenology of small-scale turbulence". Annu. Rev. Fluid Mech. Vol. 29, pp. 435-472. 1997.

[4] U. Frisch. "Turbulence: the legacy of A. N. Kolmogorov". Cambridge University Press. Cambridge, Massachusetts, USA. 1995. 
[5] F. Anselmet, Y. Gagne, E.J. Hopfinger and A. Antonia. "High-order velocity structure functions in turbulent shear flows". Journal of Fluid Mechanics. Vol. 140, pp. 63-89. 1984.

[6] O. Boratav. "On recent intermittency models of turbulence". Physics of Fluids. Vol. 9, Issue 5, pp. 1206-1208. 1997.

[7] S. Kurien and K.R. Sreenivasan. "Dynamical equations for high-order structure functions, and a comparison of a mean-field theory with experiments in three-dimensional turbulence". Phys. Rev. E. Vol. 64, pp. 056302:1-14, 2001.

[8] T. Gotoh, D. Fukayama and T. Nakano. "Velocity statistics in homogeneous steady turbulence obtained using a high-resolution direct numerical simulation". Physics of Fluids. Vol. 14. pp. 1065-1081. 2002.

[9] C. Rosales. "Synthetic three-dimensional turbulent passive scalar fields via the minimal Lagrangian map". Physics of Fluids. Vol. 23, pp. 075106-1, 075106-22. 2011.

[10] E. Novikov. "Infinitely divisible distributions in turbulence". Physical Review E. Vol. 50, Issue 5, pp. R3303-R3305. 1994.
[11] T. Halsey, M. Jensen, L. Kadanoff, I. Procaccia and B. Shraiman. "Fractal measures and their singularities: the characterization of strange sets". Physical Review A. Vol. 33, Issue 2, pp. 1141-1151. 1986.

[12] K. Sreenivasan. "Fractals and multifractals in fluid turbulence". Annual Review of Fluid Mechanics. Vol. 23, pp. 539-604. 1991.

[13] C. Meneveau and K.R. Sreenivasan. "The multifractal nature of turbulent energy dissipation". Journal of Fluid Mechanics. Vol. 224, pp. 429-484. 1991.

[14] K.R. Sreenivasan and P. Kailasnath. "An update on the intermittency exponents in turbulence". Physics of Fluids A. Vol. 5, Issue 2, pp. 512514. 1993.

[15] C. Rosales and C. Meneveau. "Anomalous scaling and intermittency in three-dimensional synthetic turbulence". Physical Review E. Vol. 78, pp. 016313:1-18. 2008.

[16] C. Rosales. "Synthetic three-dimensional turbulent passive scalar fields via the minimal Lagrangian map". Physics of Fluids. Vol. 23, pp. 075106-1, 075106-22. 2011. 